\title{
Das Gesuch
}

\section{Eine Kurzgeschichte}

A. J. Koemeda
Korrespondenz:

Dr. med. A. J. Koemeda «Breitenstein»

CH-8272 Ermatingen
Seit vier Jahren gehört unser Institut zu den besten Steuerzahlern der Gemeinde, beschäftigt mehr als dreissig Mitbürger und trägt zum guten Ruf unser ländlichen, aber touristisch wichtigen Gegend bei. Aus diesem Grund ist meinen Mitarbeitern und mir völlig unverständlich, warum unser Baugesuch «Neues Stockwerk und Hausvergrösserung» diskussionslos und, wie Sie schreiben, «mit grosser Mehrheit» abgewiesen wurde. Ich kann es mir nur so erklären, dass Sie, meine sehr geehrten Damen und Herren, über unser Institut, dessen Präsident ich nun seit einiger Zeit bin, zu wenig informiert sind. Deshalb ist es für mich wichtig, Ihnen unser Schulungszentrum und dessen Zielsetzungen kurz vorzustellen.

VOF ist die Abkürzung von «Vorbereitungsseminar für optimale Feriengestaltung». Vor sechs Jahren, zur Zeit der Gründung dieses gemeinnützigen Vereins, gingen wir von der bedenklichen Tatsache aus, dass der moderne Mensch immer mehr Mühe hat, seine Ferienzeit erholsam, gesund und umweltfreundlich zu gestalten. Eine Ferienhilfe oder, wenn Sie wollen, ein Ferienbeistand tut not, und es bedarf einer gewissen Wachsamkeit und Anstrengung, wenn der Urlaubsverlauf nicht restlos von den psychologisch zu wenig geschulten Animateuren bestimmt werden soll - ganz abgesehen davon, dass diese «Helfer» in erster Linie die Belange der Hotelbesitzer und der Souvenirladeninhaber im Auge haben, weniger aber das Wohl der Erholungssuchenden selbst.

Vor gewissen Fakten sollten wir nicht die Augen verschliessen: Eine optimale Ferienverbringung ist heute tatsächlich nicht so einfach, wie vor zwanzig oder dreissig Jahren. Dort, wo man früher mit laienhaften, ja, zum Teil auch naiven Urlaubsvorstellungen relativ gut durchgekommen ist, entstehen zu unserer Zeit komplexe Verstrickungen und Probleme, die dementsprechend vielschichtiger Lösungsstrategien bedürfen.

Ich möchte Ihre Zeit nicht mit detaillierten Überlegungen beanspruchen; zu Ihrer Orientierung erlaube ich mir, nur zwei Beispiele zu nennen, damit Sie sich ein plastischeres Bild von den Aufgaben unseres Hauses machen können:
Es gibt arme Länder, es gibt reiche Länder unerfreulich und ungerecht, zweifelsohne, aber wahr; diesen Sachverhalt vermögen wir nicht zu verändern, wir müssen ihn akzeptieren - Faktum Nummer eins.

Faktum Nummer zwei: Die wichtigsten Ströme des Urlaubsverkehrs fliessen, vor allem im Sommer, in Richtung arme Gegenden.

Faktum Nummer drei: In diesen armen Ländern herrschen andere hygienische Verhältnisse als bei uns, und das Ergebnis heisst: Erkrankung der Verdauungsorgane.

Sehr geehrte Damen und Herren, Hand aufs Herz: Welchen Erholungswert vermögen wir einer Freizeit abzugewinnen, in der wir leiden? Von welchen Regenerationsqualitäten können wir noch sprechen, wenn wir - geschwächt, blass, frische Unterwäsche schon längst verbraucht - nicht die Schönheiten des fremden Landes und die Eigenart seiner Bevölkerung wahrzunehmen im Stande sind, sondern, mit Diarrhöeproblemen fast pausenlos beschäftigt, nur an das Zuhause denken, an unsere saubere, nach Fichtennadeln duftende Intimräumlichkeit mit einwandfrei funktionierender Spülung und einer geblümten Feuchttüchleinschachtel?

Bitte, keine falschen Mutmassungen, mit einer feindlichen Einstellung unterentwickelten Ländern gegenüber hat meine Faktendarstellung nicht das geringste zu tun, wer dies behaupten will, hat alles missverstanden. Wir suchen doch nur Wege und sinnvolle Massnahmen, um unseren Kunden, den zukünftigen Urlaubsanwärtern, zu helfen, die Ferienzeit tatsächlich gesund, erholsam und kreativ zu gestalten.

Wie dies im Falle meines eingangs genannten gastro-intestinalen Beispiels konkret geschieht? Hier nur ein Hinweis, der den allgemeinen Rahmen unserer Hilfeleistung andeuten soll, ohne sich allzu ausführlich in die rein medizinische Seite zu vertiefen:

Unsere Urlaubsanwärter durchlaufen, was das Essen anbelangt, drei Verpflegungsstufen, wobei sich die einzelnen Phasen im Grad der bakteriellen Reinheit unterscheiden. Auf Stufe 1 (sechs bis zehn Tage) bekommt man praktisch herkömmliche Ernährung, die nur mit geringen Mengen von Bakterium-Coli-Konzentraten verunreinigt sind. In der zweiten Etappe, in der ei- 
gentlichen Gewöhnungsphase (sieben bis vierzehn Tage), werden die pathogenen Zutaten kontinuierlich gesteigert, und zwar bis zum Auftreten der üblichen Dickdarmsymptomatik von subklinischer Ausprägung («Flotter Otto» wird dieses Stadium von unseren Klienten salopp genannt). Auf der dritten Stufe (drei bis sechs Tage) wird dem Baldurlauber Kost vorgelegt, die, was den Grad der bakteriellen Verseuchung anbelangt, es mit den Speisen des jeweiligen Ferienlandes aufnehmen kann - sofern die Wahl des Hotels nicht auf ein Viersternetablissement fällt und man keine risikoreichen Besuche einheimischer Lokale plant.

Haut, Verdauungsorgane, respiratorischer Trakt ... praktisch alles, womit unser Körper reagieren könnte, wird bedacht und berücksichtigt. Damit soll aber nicht gesagt werden, dass wir rein technisch-medizinisch vorgehen und dem Körperlichen Vorrang geben. Nein, das keineswegs!

Das zweite Beispiel kann Ihnen zeigen, wie bei uns die Vorbereitungen auf ideeller Ebene mit den rein medizinischen Schritt halten - ohne als zweitrangig betrachtet zu werden.

Hier möchte ich mit der Beschreibung eines Bildes anfangen, eines farbigen Fotos, das ich vor wenigen Wochen in einer deutschen Illustrierten sah. Ein dunkelhäutiger Junge steigt aus einer felsigen Höhle und lächelt verlegen. In seinen Armen trägt er ein mit braungrauer Haut überzogenes Skelett, eine Mumie. Die Mumie eines seiner Vorfahren! Der Begleittext zu diesem doppelseitigen Bild: «Die Ruhe der Ahnen ist den philippinischen Bergvölkern heilig, aber für ein paar Dollar findet sich jemand, der seine Angst vor der Rache der Geister überwindet.»

Jawohl, dieser Junge, etwa zwölf, dreizehn Jahre alt, überwand seine Angst vor dem Zorn der Toten, stieg in das dunkle Erdloch und brachte den eigenen Urgrossvater ans Tageslicht, um ihn fotografieren zu lassen, um den sensationslüsternen Touristen einige Cendaros abzunehmen, um ein paar Münzen nach Hause zu bringen.

Ist das in Ordnung, ist das richtig so? Die Frage ist überflüssig. Ich will nur sagen, schlimm ist die Entwicklung in den letzten Jahren, wirklich schlimm - sterbende Kulturen freizugeben zum Schnappschuss!
Aber wo - und hier muss ich, sehr geehrte Damen und Herren, auch Ihnen die Frage vorlegen - wo kann der heutige Mensch das richtige Ferienverhalten erlernen, die Tabugrenzen erkennen und Anstand praktizieren? Wer bringt ihm die Regeln bei, den selbstkritischen Blick, den Respekt vor dem Fremdartigen, die notwendige Zurückhaltung? Die Antwort lautet eindeutig: Niemand!

Jawohl, niemand. Bis jetzt zumindest!

Seit der Gründung des VOF hat sich die Situation für den Erholungssuchenden schlagartig verändert. Dem Urlaubsanwärter steht bei uns eine ganze Reihe von Ferienpsychologen zur Seite, die bereit sind, ihr theoretisches Wissen und ihre praktischen Facherfahrungen zur Verfügung zu stellen. Die Akzente unserer Arbeit liegen gleichgewichtig verteilt - sowohl auf der rein körperlichen Einstimmung bzw. der physischen Ertüchtigung des Klienten als auch auf der Information, sozialhumanistischen Erziehung und reisepsychologischen Urlaubsvorbereitung. Denn: Eine gesund und sinnvoll verbrachte Ferienzeit ist nichts anderes als ein optimal geratenes psychosomatisches Kunstwerk.

Wir, und hier möchte ich im Namen aller VOF-Mitarbeiter sprechen, wir sind fest davon überzeugt, dass eine fachlich einwandfrei geführte Ferienschulung bald fester Bestandteil des Unterrichtsplanes sein wird, und zwar nicht nur an den höheren Lehranstalten, sondern auch an den kantonalen Primar- und Sekundarschulen. Die Zeit des «Amateururlaubers», des «Ferienwilderers» wie auch des «Erholungsgeschädigten» wird dann endgültig dem grauen Abschnitt des vorhumanen Reisezeitalters angehören.

In der Hoffnung, sehr verehrten Damen und Herren, dass Ihnen diese Ausführungen helfen werden, das Anliegen des VOF besser zu verstehen, erlaube ich mir, dieses Schreiben als Begleitbrief meines Rekurses beizulegen.

Im Namen des VOF-Vorstandes verbleibe ich mit freundlichen Grüssen

Hannes Mario Sonne 